\title{
Ezrin in primary cutaneous melanoma
}

\author{
Suvi Ilmonen ${ }^{1}$, Antti Vaheri ${ }^{2}$, Sirpa Asko-Seljavaara ${ }^{1}$ and Olli Carpen ${ }^{3}$ \\ ${ }^{1}$ Department of Plastic Surgery, Helsinki University Hospital, Helsinki, Finland; ${ }^{2}$ Department of Virology and \\ ${ }^{3}$ Pathology and Anatomy and Neuroscience Program, Biomedicum, University of Helsinki, Helsinki, Finland
}

\begin{abstract}
Ezrin is a member of the ezrin-radixin-moesin family of proteins that link the actin-containing cytoskeleton to the plasma membrane. Ezrin is also connected to signaling molecules involved in the regulation of cell survival, proliferation and migration. Here, we examined the expression of ezrin in 95 primary cutaneous melanomas and correlated ezrin expression with conventional prognostic factors and biomarkers. From 12 patients metastatic tissue samples were also examined. In addition to ezrin staining, Mib-1 proliferation antigen, p53 and Bcl-2 were evaluated. Ezrin immunoreactivity was seen in most tumors; only $19(20 \%)$ melanomas were negative. A total of $48(51 \%)$ tumors had weak immunoreactivity and $28(29 \%)$ strong immunoreactivity. The intensity of ezrin immunoreactivity was associated with tumor thickness (Breslow, $P=0.0008$ ) and with tumor invasion level (Clark, $P=0.004)$, thicker tumors having stronger immunoreactivity. Also, there was a correlation between higher Mib-1 index in tumors and strong ezrin expression. All metastatic samples $(n=12)$ showed positive ezrin immunoreactivity. In univariate analysis of survival, patients $(n=76)$ with positive ezrin immunoreactivity had worse clinical disease behavior than those $(n=19)$ without ezrin immunoreactivity, but the difference was not significant $(P=0.19)$. In multivariate analysis of survival, the ezrin immunoreactivity was not a significant marker. The results indicate that ezrin is expressed in most primary melanomas of the skin and in all metastatic tumors. Ezrin expression correlates with tumor thickness and level of invasion suggesting an association between ezrin expression and tumor progression.
\end{abstract}

Modern Pathology (2005) 18, 503-510, advance online publication, 8 October 2004; doi:10.1038/modpathol.3800300

Keywords: ezrin; ERM protein; immunohistochemistry; melanoma; metastasis; prognostic factor

The most important prognostic marker of primary cutaneous melanomas is tumor thickness (Breslow classification). ${ }^{1,2}$ Tumor ulceration, an adverse prognostic sign, is the second strongest independent predictor of outcome in localized disease and is also significant in regional, that is, nodal disease. ${ }^{2,3}$ The depth of invasion (Clark classification) is a significant factor only in thin melanomas, where it is included in the new staging system. ${ }^{4,5}$ Clinical features such as patient's age and gender, and location of the tumor are of minor prognostic importance. ${ }^{1,2,4,6}$ Dissemination of the tumor to sentinel node, the first lymph node in the lymph basin draining from the tumor, appears to be the single most important factor in prediction of the disease outcome. ${ }^{2,7}$

All these markers are useful in defining the surgical and oncological treatment of melanoma patients. However, there is a variation in the outcome of patients within each Breslow score or

Correspondence: Dr S Ilmonen, MD, Department of Plastic Surgery, Töölö Hospital, PO Box 266, Helsinki University Hospital, 00029 Helsinki, Finland.

E-mail: suvi.ilmonen@hus.fi

Received 22 June 2004; revised and accepted 26 August 2004; published online 8 October 2004 within sentinel node positive individuals. Some superficial melanomas (Breslow score $<1.0 \mathrm{~mm}$ ) behave aggressively and metastasize early, ${ }^{8,9}$ and some patients with thick melanoma do surprisingly well surviving longer than expected, suggesting that there are unknown biological factors contributing to disease progression. Identification and understanding of the functions of such factors would be crucial for improved treatment of melanoma patients and for development of novel therapeutic modalities.

Numerous melanoma markers have been studied on tissue by immunohistochemical methods. They can be categorized according to the basis of their biological function: (1) markers of cellular proliferation, Ki-67 and proliferation cell nuclear antigen (PCNA), (2) cell cycle regulators, p53, p16 and cyclin D1, (3) extracellular matrix-degrading enzymes, plasminogen activators and matrix metalloproteinases, (4) cell adhesion molecules, integrins, CD44, Mel-CAM/MUC18 and ICAM-1. Also, telomerase activity and microphthalmia-associated transcription factor (Mitf) are among new markers with potential prognostic value. ${ }^{6,10-12}$ Besides immunohistochemical markers, there is a variety of serological melanoma markers that are secreted to blood either directly by melanoma cells or as an indirect response of the patient's immune system. ${ }^{13}$ 
Mib-1, a proliferation marker, which recognizes the Ki-67 antigen, is expressed at increased levels both in primary and metastatic stages of melanoma. Some studies suggest that Ki-67 expression in primary tumors can be useful as an independent prognostic factor. These cohorts have mainly consisted of thick (at least $>1.5 \mathrm{~mm}$ ) tumors-an evaluation of thinner tumors has not been properly assessed. ${ }^{10,14-17}$ p53 is a tumor suppressor protein that has an inhibitory function in cell cycle progression. With this capacity, it allows cells to repair DNA defects, thus preventing the proliferation of mutated cells. p53 can also initiate apoptosis if the damaged DNA is not repaired. Mutations of the $p 53$ gene are common in malignancies leading to a production of a mutated p53 protein that may lack the capacity to hinder the cell cycle. The role of p53 mutations in the prognosis of melanoma remains controversial. ${ }^{15,18-21}$ Bcl-2, an antiapoptotic protein, may have a role in tumor development by sparing cells from apoptotic death and thus exposing them to secondary events. Bcl-2 is expressed in primary and metastatic melanomas but results on its prognostic role are inconsistent. ${ }^{22-24}$

Ezrin, a protein of molecular weight of about $70 \mathrm{kDa}^{25,26}$ is a member of the ezrin-radixin-moesin family of proteins, which link the actin-containing cytoskeleton to the plasma membrane. ${ }^{27-29}$ They bind to cell surface glycoproteins such as CD43, CD44 and ICAM-1 and -2 by their amino (N)terminal domains and to filamentous actin by their carboxy (C)-terminal domains. Ezrin possesses morphogenic properties and has a role in regulation of cell adhesion and migration. ${ }^{30,31}$ In addition to membrane proteins and F-actin in cortical cytoskeleton, ${ }^{32}$ ezrin is linked to molecules that control the phosphatidylinositol-3-kinase, ${ }^{33}$ protein kinase $\mathrm{A}^{34}$ and Rho pathways. ${ }^{35}$ Ezrin is thus functionally involved in signaling events that regulate cell survival, cell proliferation and migration..$^{28,29,31}$ In melanoma cells, ezrin has been localized in phagocytic vacuoles, suggesting that the association with the actin cytoskeleton is a crucial requirement for the phagocytic activity. Phagocytic behavior is further considered as an indicator of high-grade malignancy in melanomas. ${ }^{36}$ Interestingly, merlin, a close relative of ezrin, is a tumor suppressor protein inactivated in neurofibromatosis type 2 , a disease that predisposes to multiple nervous system tumors. $^{37}$

Ezrin is expressed in a variety of normal and neoplastic cells, including many types of epithelial, lymphoid and glial cells. ${ }^{28}$ Studies have suggested a role for ezrin in the in vivo behavior of several tumor types. Immunohistochemical analysis demonstrated a significant correlation between increased ezrin immunoreactivity and higher histological grade in astrocytoma. $^{38}$ The association between ezrin immunoreactivity and malignancy in these tumors was stronger than the correlation between the proliferation marker Ki-67/MIB-1 and malignancy. In a complementary DNA (cDNA) microarray analysis of highly and poorly metastatic rhabdomyosarcoma, ezrin was indicated as a key metastatic regulator. ${ }^{39}$ In a mouse osteosarcoma model, suppression of ezrin protein and disruption of ezrin function significantly reduced lung metastases in mice. ${ }^{40}$ Furthermore, high ezrin expression in dog osteosarcomas was associated with early development of metastases. Consistently in the same study, a small set of prospectively collected tissues from pediatric patients with localized osteosarcoma was examined. Patients with high ezrin tumor staining had a significantly shorter disease-free interval compared with patients with low ezrin staining. ${ }^{40}$ In serous ovarian carcinomas, on the other hand, the correlation with ezrin and prognosis was more complex, tumors not expressing ezrin having the worst prognosis. ${ }^{41}$

An earlier study showing an association between increased ezrin immunoreactivity and increased mortality in uveal melanoma ${ }^{42}$ prompted us to study ezrin expression and its prognostic significance in primary cutaneous melanomas, which represents a much more common patient population. We also wanted to study, whether ezrin expression correlates with prognostic markers in clinical use and with commonly used biological markers such as Mib-1, Bcl-2 and p53. In a subset of patients, we compared ezrin immunoreactivity in primary and metastatic tumors.

\section{Materials and methods}

\section{Study Material}

A total of 95 patients with a primary melanoma of the skin were included in the study. This patient population represents a randomly selected subgroup from a clinical series that includes all primary melanoma patients treated at the Department of Plastic Surgery, at Helsinki University Hospital, Finland, during the years 1988-1991. The melanoma diagnosis, tumor thickness and the level of tumor invasion were re-evaluated from the slides. Ulceration characteristics were coded positive if reported so in the original microscopical examination. During the period of primary diagnosis, the presence of ulceration was not routinely included in the pathology report; therefore the number of ulcerative tumors may be underestimated. From 12 patients, the first metastatic tissue specimen was also analyzed. Clinical characteristics of the patient population and the tumors are presented in Table 1.

Patients were followed up postoperatively at 3-month intervals for the first 2 years, and thereafter biannually up to 5 years. Radiological examinations and fine-needle biopsies were conducted on the basis of the clinical status. The clinical follow-up for recurrences and metastases lasted for 0.15-10.0 years (median 4.8 years), and for overall survival 
Table 1 Patient and tumor characteristics

\begin{tabular}{llr}
\hline Patients & & $\mathrm{n}=95(\%)$ \\
\hline \multirow{2}{*}{ Gender } & Male & $44(46)$ \\
Age & Female & $51(54)$ \\
Tumor thickness & Median 58.6 years & Range (24.0-84.6) \\
Depth of tumor invasion & Median 1.20 mm & Range (0.40-15.60) \\
& Clark I & $1(1)$ \\
& Clark II & $7(7)$ \\
& Clark III & $50(53)$ \\
& Clark IV & $32(34)$ \\
Tumor location & Clark V & $4(4)$ \\
& Unclassified & $1(1)$ \\
& Head or neck & $11(12)$ \\
& Trunk & $36(38)$ \\
& Upper extremity & $17(18)$ \\
& Palm & $1(1)$ \\
Ulceration & Lower extremity & $27(28)$ \\
& Sole & $3(3)$ \\
& Positive & $15(16)$ \\
& Negative & $80(84)$ \\
\hline
\end{tabular}

0.5-15.4 years (median 9.1 years). The clinical follow-up data were collected from patient records and the overall survival thereafter was obtained from the National Population Registry.

\section{Ezrin Immunohistochemistry}

Sections of $5 \mu \mathrm{m}$ thickness were cut from paraffinembedded tissue blocks. Immunostaining for ezrin was performed using the avidin-biotin peroxidase complex method (UltraVision, Lab Vision Inc., Fremont, CA, USA). The primary murine IgG monoclonal antibody (mAb) to human ezrin (clone 3C12, ascites, diluted 1:2000) ${ }^{43}$ was used after antigen retrieval in $10 \mathrm{mM}$ sodium citrate buffer (pH 6.0) for $10 \mathrm{~min}$ at $95^{\circ} \mathrm{C}$. The $\mathrm{mAb}$, raised against the C-terminal part of ezrin (amino acids 362-585), detects a single protein band compatible with ezrin in immunoblotting and does not crossreact with moesin or radixin. The slides were stained using a LabVision Autostainer automatic immunostaining device (Lab Vision Inc.). An unrelated murine IgG mAb (clone X63, ascites, diluted 1:1000; American Type Culture Collection, Manassas, VA, USA) served as a negative control.

All slides, both primary and metastatic, were scored independently by two investigators (OC and SI) blinded to patient outcome. In case of discrepancy, a consensus was formed. The immunoreactivity was initially scored as a four-category variable; negative (immunoreactivity 0), weak (immunoreactivity 1), intermediate, and strong expression of ezrin (Figure 1). Since the groups with intermediate or strong immunoreactivity remained small, they were grouped (immunoreactivity 2) for all analyses. The epithelial cells of surrounding normal skin and the peritumoral lymphocytes served as a reference of strong immunoreactivity.

\section{Bcl-2, Mib-1 and p53 Immunohistochemistry}

Bcl-2, Mib-1 and p53 were immunostained using VESTASTAIN $^{\circledR}$ Elite ABC KIT (Vector Laboratories, Inc., Burlingame, CA, USA) immunoperoxidase technique. After antigen retrieval, the slides were reacted with anti-Bcl-2 mAb, diluted 1:200 (Dako, Glostrup, Denmark), anti-p53 mAb, diluted 1:300 (Dako) or anti-Mib-1 mAb, diluted 1:500 (Immunotech, Marseilles, France). Incubation with primary antibody was carried out overnight in a moist chamber at room temperature. The slides were then incubated with biotinylated secondary antibody solution, rinsed, and incubated for $30 \mathrm{~min}$ with VECTASTAIN $^{\circledR}$ Elite ABC Reagent. 3-Amino-9ethylcarbazole (AEC, Sigma A-5754) solution $(0.2 \mathrm{mg} / \mathrm{ml}$ AEC in $0.005 \mathrm{M}$ acetate buffer that contains $0.03 \%$ perhydrol in $\mathrm{pH} 5.0$ ) was used as chromogene for $15 \mathrm{~min}$ at room temperature. Mayer's hematoxylin was used for counter staining. Slides were mounted with Aquamount (BDH Ltd, Poole, UK).

Of 95 primary tumors, 75 were immunostained for Bcl-2, 80 for Mib-1 and 71 for p53. The rest were left out because of running out of tumor tissue or technical problems. The slides were examined independently by two readers (SI and Micaela Hernberg), and in case of discrepancy, the slides were reanalyzed and an agreement reached. In analysis of Bcl-2, the entire tumor area was evaluated and the result reported as percentage of positively stained tumor area. In analysis of p53 and Mib-1, the proportion of positive cells among 200 tumor cells was reported.

\section{Statistical Analysis}

Melanoma-related disease-free survival time was calculated as the time interval between diagnosis and first recorded sign of disease progression after primary operation. Melanoma-related overall survival was defined as the time interval between diagnosis and death of melanoma. Patients with no signs of progression at last follow-up visit and those patients who died of other causes were censored at the time of the last visit or death, respectively.

All analyses were performed with Statview ${ }^{\mathrm{TM}}$ statistical program. The distribution of the measured parameters was characterized by standard descriptive methods. Contingency tables were analyzed by $\chi^{2}$ test and Fisher's exact test. The nonparametric Mann-Whitney $U$-test, Kruskal-Wallis test and Spearman rank correlation test were used for analyzing nonparametric variables. Univariate analysis of disease-free and disease-specific overallsurvival was based on Kaplan-Meier method, and differences in survival were tested with Mantel-Cox (log-rank) test. Stepwise Cox multivariate analysis was used to identify independent prognostic factors. $P$-values $\leq 0.05$ were regarded as statistically significant. 

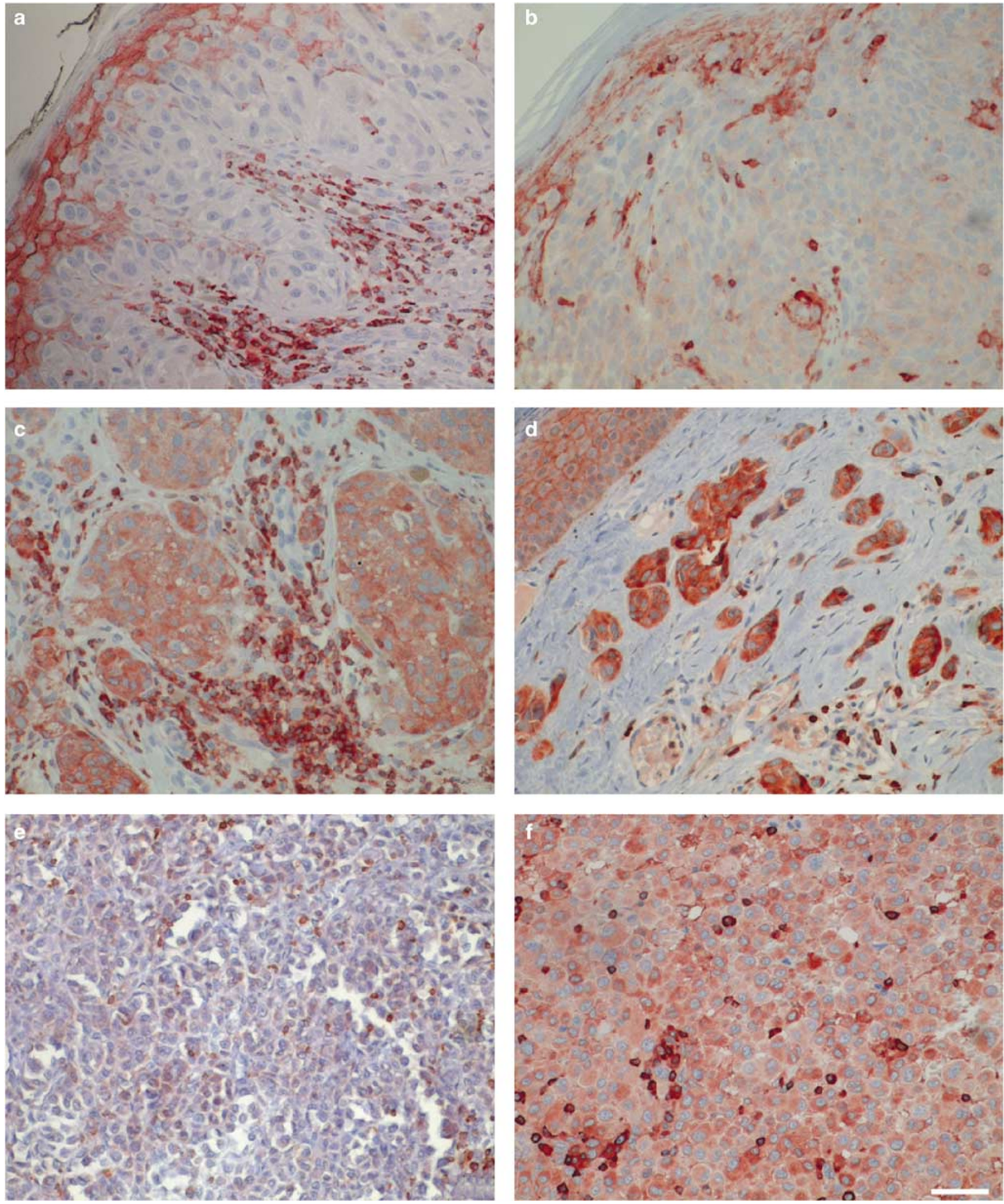

Figure 1 Ezrin immunoreactivity in melanoma specimens. Examples of tumors with different staining intensities are shown. (a) Negative ezrin immunoreactivity (0); (b) weak ezrin immunoreactivity (1); (c) intermediate ezrin immunoreactivity (2); (d) strong ezrin immunoreactivity (2); (e-f) immunoreactivity in primary melanoma (e) and in a metastatic tumor from the same patient (f). In upper left corner of (a, b and $\mathbf{d}$ ) staining of the basal layers of the epidermis is seen. Note also strong immunoreactivity of tumor-associated lymphocytes in (a) and (c). 


\section{Results}

Ezrin Immunoreactivity in Primary Melanoma and Metastatic Lesions

Ezrin immunostaining with $3 \mathrm{C} 12 \mathrm{mAb}$ was successful in all 95 primary melanomas. Tumor cell staining was encountered in most (76/95) tumors. In all specimens, basal epidermal cells of the adjacent normal skin and the surrounding lymphocytes demonstrated strong ezrin immunoreactivity, thus providing an internal control of positive staining. In total, 19 melanomas were classified as negative (immunoreactivity 0), 48 as weakly positive (immunoreactivity 1), and 28 as positive with strong intensity (immunoreactivity 2). When positive, the immunoreaction tended to be homogenous throughout the tumor; however, in a few tumors, the intensity varied in different regions of the tumor. In these cases, higher intensity staining of $\geq 20 \%$ justified classification to the higher category.

Ezrin immunoreactivity of metastatic tumors was in general stronger than in primary tumors. None of the metastases were negative for ezrin, two samples showed weak immunoreactivity and 10 strong immunoreactivity. In six cases, the intensity of metastatic tumor was higher than in primary tumor (prim 0-met 1, $n=1$; prim 1-met 2, $n=5$ ) and in six tumors, the intensity remained unchanged (prim $1-$ met $1, n=1$; prim $2-$ met $2, n=5$ ). In none of the tumors, the intensity of primary tumor was higher than in metastasis.

\section{Association between Ezrin Immunoreactivity and Other Tumor Characteristics}

The level of ezrin immunoreactivity did not correlate with patient's gender, age, tumor ulceration or location. However, a strong positive correlation with tumor thickness $(P=0.0008$, Breslow classification) and the level of tumor invasion $(P=0.004$, Clark classification) was seen (Figure 2a and b). The number of tumors with ezrin immunoreactivity 2 correlated directly and the number of tumors with ezrin immunoreactivity 0 correlated inversely with increased tumor thickness and invasion levels, whereas the proportion of ezrin immunoreactivity 1 tumors was not significantly influenced by tumor thickness. There was a correlation between higher Mib-1 index and increased ezrin immunoreactivity $(P=0.04)$. Mib-1 index had a weak correlation to tumor thickness (Breslow classification, $P=0.10$ ), and a stronger association to the level of tumor invasion (Clark classification, $P=0.009$ ); Mib-1 being higher in thicker tumors. With respect to Bcl-2, our study showed no correlation between ezrin immunoreactivity and Bcl-2 expression. Neither was any correlation seen between ezrin immunoreactivity and p53 expression.
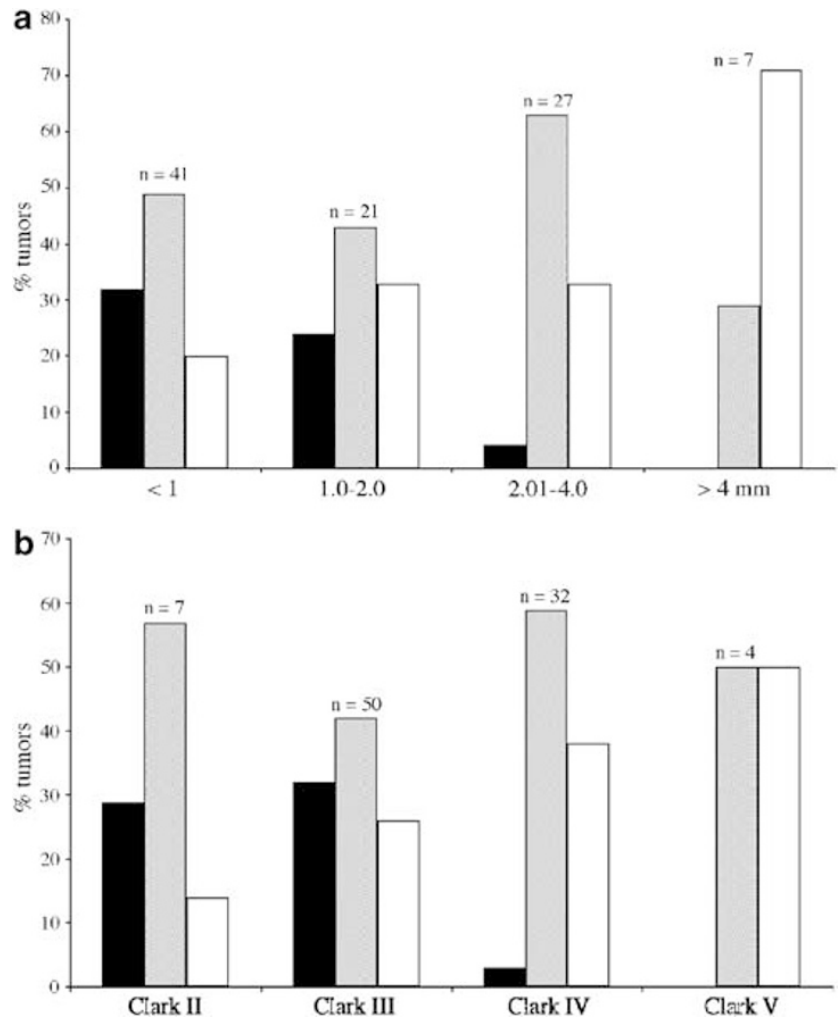

Figure 2 Correlation between ezrin immunoreactivity and melanoma thickness or invasion level. (a) Tumors were categorized according to the Breslow classification. Intensity of ezrin immunoreactivity increased with tumor thickness $(P=0.0008)$. (b) Ezrin immunoreactivity was compared with Clark classification. Increased ezrin staining was seen in higher Clark categories $(P=0.004)$. White bar $=$ immunoreactivity 0 , gray bar $=$ immunoreactivity 1 , black bar $=$ immunoreactivity $2 ; n=$ number of tumors in each category.

\section{Ezrin and Clinical Outcome}

At the end of the follow-up, $26(27.4 \%)$ patients had had a recurrent melanoma, and $16(16.8 \%)$ had died of it. In all, 20 patients $(21.1 \%)$ had died of other reasons, $59(62.1 \%)$ were alive at the end of followup. For patients with tumors with absent ezrin immunoreactivity, the melanoma-specific probability of overall-survival was $89.5 \%$, for patients with tumors with weak ezrin immunoreactivity it was $77.1 \%$, and for those with tumors with strong ezrin immunoreactivity, it was $89.3 \%$. In disease-free survival, the probability for surviving without melanoma recurrences or metastases was $89.5 \%$ in the group of patients with tumors with absent ezrin immunoreactivity, $68.8 \%$ with tumors with weak immunoreactivity, and $67.9 \%$ with tumors with strong ezrin immunoreactivity.

In univariate analysis for disease-free survival, independent prognostic factors were tumor thickness $(P=0.0002)$, tumor invasion level $(P=0.02)$, and p53 staining $(P=0.007)$. In analysis of p53, the division into two groups was made at the mean 
value of $7.3 \%$; those below that value had a survival benefit. In univariate analysis of melanoma-specific overall survival, independent prognostic markers were tumor thickness $(P=0.003)$, tumor invasion level $(P=0.04)$, age $(P=0.006)$ and ulceration $(P=0.05)$. Younger patients had a survival benefit, and tumor ulceration was an adverse prognostic marker. Neither Mib-1 nor Bcl-2 had any prognostic value.

With regard to ezrin characteristics, the greatest difference in survival was detected between tumors with absent ezrin immunoreactivity and with positive immunoreactivity (either weak or strong). In univariate analysis, patients with absent ezrin immunoreactivity had a disease-free survival benefit $(2 / 19,10.5 \%$, with a metastatic disease) compared to those with positive ezrin immunoreactivity $(22 / 76$, $28.9 \%$, with a metastatic disease); however, the difference was not significant $(P=0.19)$. For melanoma-specific overall survival, there were no statistical differences between tumors with or without ezrin immunoreactivity.

For multivariate analysis of survival, we used ezrin as a two-category variable; melanomas with absent ezrin immunoreactivity and melanomas with ezrin immunoreactivity. Other parameters were: tumor thickness (Breslow classification), age at diagnosis, ulceration characteristics and p53. For disease-free survival, tumor thickness was an independent prognostic marker $(P=0.02)$. p53 was an additional independent prognostic factor $(P=0.03)$, low p53 value indicating better prognosis. For melanoma-specific overall survival age was the strongest prognostic marker $(P=0.02)$. Age below median, 58.6 years, was a favorable prognostic indicator. An additional independent prognostic marker was tumor thickness $(P=0.04)$.

\section{Discussion}

In this study, we examined ezrin expression and its correlation with clinical outcome in primary cutaneous melanoma. The intensity of ezrin immunoreactivity was found to increase with tumor size, as measured by tumor thickness (Breslow classification) and invasion to dermal layers (Clark classification). In both instances the correlation was statistically significant. The intensity of ezrin immunoreactivity also correlated with tumor proliferation index, Mib-1. In tumors with ezrin immunoreactivity 0, Mib-1 expression was low, whereas in tumors with ezrin immunoreactivity 1-2, Mib-1 expression was clearly enhanced.

In line with the results on uveal melanomas and osteosarcomas, ${ }^{40,42}$ our study suggests that ezrin may have a role in tumor progression and disease outcome. The biological role of ezrin in the cascade of tumor growth and dissemination is not yet clear but several possibilities exist. Ezrin has an important role in cell adhesion and cell-cell communica- tion. E-cadherin-associated cell-cell interactions and integrin-mediated interactions with the extracellular matrix are thought to be important in metastatic progression. ${ }^{44,45}$ Ezrin is also necessary for several signaling pathways, that is, MAPK, Akt, CD44, Rho kinase and HGF, identified as tumor metastasis-associated proteins. ${ }^{40,46,47}$ Recio et al showed in their study that 37-32 murine melanoma cells possess a strong HGF/SF-Met autocrine loop and express the variant 6 isoform of CD44. Further, CD44v6 variant is upregulated by HGF/SF through a feedback loop requiring the presence of early gene Egr-1. These mechanisms are important for the maintenance of the stimulation of metastasis-promoting pathways in melanoma cells. ${ }^{48}$ The rhabdomyosarcoma study on mouse cell lines suggested a correlation between ezrin expression and Rho activity. Overexpression of ezrin induced highly metastatic state in poorly metastatic rhabdomyosarcoma cell lines, the prometastatic activity of ezrin being at least partly mediated through Rho. ${ }^{39}$ In the study of Khanna et $a l,{ }^{40}$ suppression of ezrin resulted in decreased signaling and activity of Akt and p44/42 mitogen-activated protein kinase (MAPK) pathways in highly metastatic osteosarcoma cells. Further, survival of osteosarcoma cells was partially rescued by overexpression of activated p44/42 MAPK, but not Akt, suggesting that ezrinmediated cell survival either bypasses Akt or operates through another anti-apoptotic pathway. ${ }^{40}$ MAPK signaling pathway that regulates cellular differentiation, proliferation and survival has been previously studied in melanoma. ${ }^{49}$ Koo et $a 5^{50}$ showed that inhibition of MAPK signaling in melanoma cells induced apoptosis suggesting a direct role for MAPK in signaling melanomagenesis and survival. The Ras-Raf-MAPK pathway is activated in the majority of melanomas because of a mutation in the BRAF gene. ${ }^{51}$ In a mouse melanoma model, pharmacological inhibition of this pathway was a promising target for therapy. Thus, enhanced ezrin expression in melanoma cells might contribute to increased MAPK levels and thus influence tumor cell proliferation and metastasis.

Rather than influencing one single intracellular event, ezrin is involved in many intracellular signaling processes and in cell-adhesion functions. The high expression of ezrin in primary tumor may directly contribute to metastasis, or it can be a marker of a tumor cell that is responding to growth factors, adhesion molecules and other signaling pathways that are dependent on ezrin. ${ }^{40}$ Little is known about the roles of radixin and moesin and their possible interactions with ezrin in tumorigenesis. Ichikawa et $a l^{52}$ reported that moesin, a member of ezrin-radixin-moesin family, was intensively expressed in benign melanocytic nevi and malignant melanoma in situ, but in invasive melanoma and in skin and lymph node metastases of melanoma, the moesin immunoreactivity was reduced. 
Ezrin may also be involved in the late process of tumor progression and metastasis. In the study of Ohtani et al of uterine endometrioid adenocarcinoma, tissues of primary and metastatic lesions were obtained from five patients. They showed that ezrin immunoreactivity was significantly higher in metastatic tissues than in the matched primary tumors. ${ }^{53}$ In our study, none of the metastases were devoid of ezrin, on the contrary, comparison of primary and metastatic samples of the same patients revealed that the intensity of ezrin immunoreactivity either remained similar or increased during disease progression. Akisawa et al ${ }^{54}$ showed the same phenomenon in human pancreatic adenocarcinoma cell lines, suggesting that high ezrin expression is correlated with metastatic potential.

Uveal and cutaneous melanomas represent different disease entities with different treatment methods and disease outcomes. However, similarities in ezrin expression patterns exist and correlation to clinical patient outcomes is comparable. In the study of uveal melanomas, two-thirds of melanomas were immunoreactive to ezrin, ${ }^{42}$ whereas in skin melanoma the proportion of tumors with positive immunoreactivity was even higher $(75 \%)$. In uveal melanoma, typically diffuse granular cytoplasmic immunoreaction was observed, as was the case in cutaneous melanomas; only rarely did a tumor contain distinct areas with both absent and positive ezrin immunoreactivity. In uveal melanoma, immunoreactivity for ezrin was significantly associated with microvascular density, a rough index of tumor vascularity. High microvascular density is traditionally linked with angiogenesis, hematogenous metastasis being the only way by which intraocular uveal melanoma can disseminate. The dissemination of cutaneous melanoma differs from uveal; it mainly occurs via lymphatic pathways. In the study of uveal melanoma, the patients with an ezrin-immunopositive tumor had a worse prognosis than those with negative ezrin immunoreactivity. In cutaneous melanoma, the trend was similar, but statistical significance was not reached.

In conclusion, the level of ezrin immunoreactivity was found to be associated with tumor proliferation index and tumor growth in primary melanoma of the skin. Although the result is of interest in the analysis of the molecular pathogenesis of melanoma, ezrin immunohistochemistry does not provide aid in prognostic determination of individual tumors.

\section{Acknowledgements}

We thank Tuula Halmesvaara, Tuula Nousiainen and Kristina von Boguslawski for technical support, Timo Muhonen for help in statistical analyses and Micaela Hernberg for kindly evaluating the Bcl-2, Mib-1 and p53 immunostaining. The study was financially supported by Kurt Palander Foundation,
Helsinki University Central Hospital, the Academy of Finland and the Finnish Cancer Foundation.

\section{References}

1 Breslow A. Thickness, cross-sectional area, and depth of invasion in the prognosis of cutaneous melanoma. Ann Surg 1970;172:902-908.

2 Balch CM, Houghton AN, Sober AJ, et al. Cutaneous Melanoma, 4th edn. Quality Medical Publishing: St. Louis, MO, 2003.

3 Ruiter DJ, Spatz A, van der Oord JJ, et al. Pathologic staging of melanoma. Semin Oncol 2002;29:370-381.

4 Clark Jr WH, From L, Bernandino EA, et al. The histogenesis and biologic behavior of primary human malignant melanomas of the skin. Cancer Res 1969;29: 705-726.

5 Balch CM, Soong SJ, Atkins MB, et al. An evidencebased staging system for cutaneous melanoma. CA Cancer J Clin 2004;54:131-149.

6 Rogers GS, Braun SM. Prognostic factors. Dermatol Clin 2002;20:647-658.

7 Morton DL, Wen D-R, Wong JH, et al. Technical details of intraoperative mapping for early stage melanoma. Arch Surg 1992;127:392-399.

8 Slingluff CL, Vollmer RT, Reintgen DS, et al. Lethal 'thin' malignant melanoma. Identifying patients at risk. Ann Surg 1988;208:150-161.

9 Naruns P, Nizze J, Corchan A, et al. Recurrence potential of thin malignant melanoma. Cancer 1986;57: 545-548.

10 Mangini J, Li N, Bhawan J. Immunohistochemical markers of melanocytic lesions. Am J Dermatopathol 2001;24:270-281.

$11 \mathrm{Li} \mathrm{N}$, Mangini J, Bhawan J. New prognostic factors of cutaneous melanoma: a review of the literature. J Cutan Pathol 2002;29:324-340.

12 Yaziji H, Gown AM. Immunohistochemical markers of melanocytic tumors. Int J Surg Pathol 2003;11:11-15.

13 Garbe C, Leiter U, Ellwanger U, et al. Diagnostic value and prognostic significance of protein S-100 $\beta$, melanoma-inhibitory activity, and tyrosinase/MART-1 reverse transcription-polymerase chain reaction in the folllow-up of high-risk melanoma patients. Cancer 2003;97:1737-1745.

14 Böni R, Doguoglu A, Burg G, et al. MIB-1 immunoreactivity correlates with metastatic dissemination in primary thick cutaneous melanoma. J Am Acad Dermatol 1996;35:416-418.

15 Vogt T, Zipperer K-H, Vogt A, et al. p53-protein and Ki-67-antigen expression are both reliable biomarkers of prognosis in thick stage I nodular melanomas of the skin. Histopathology 1997;30:57-63.

16 Ramsay JA, From L, Iscoe NA, et al. Mib-1 proliferative activity is a significant prognostic factor in primary thick cutaneous melanomas. J Invest Dermatol 1995; 105:22-26.

17 Hazan C, Melzer K, Panageas KS, et al. Evaluation of the proliferation marker Mib-1 in the prognosis of cutaneous malignant melanoma. Cancer 2002;95: $634-640$.

18 Agarwal ML, Taylor WR, Chernov MV, et al. The p53 network. J Biol Chem 1998;273:1-4.

19 Levine AJ, Perry ME, Chang A, et al. The 1993 Walter Hubert Lecture: the role of the p53 tumor-suppressor gene in tumorigenesis. Br J Cancer 1994;69:409-416. 
20 Weiss J, Heine M, Körner B, et al. Expression of p53 protein in malignant melanoma: clinicopathological and prognostic implications. Br J Dermatol 1995;133: 23-31.

21 Stone CH, Lynch EF, Linden MD, et al. Immunocytochemical evaluation of proliferating cell nuclear antigen, Ki-67 (Mib-1), and p53 in predicting survival of primary and metastatic malignant melanomas. Appl Immunohistochem Mol Morphol 1996;4:25-33.

22 Cerroni L, Soyer HP, Kerl H. Bcl-2 protein expression in cutaneous malignant melanoma and benign melanocytic nevi. Am J Dermatopathol 1995;17:7-11.

23 Ramsay JA, From L, Kahn HJ. Bcl-2 protein expression in melanocytic neoplasms of the skin. Mod Pathol 1995;8:150-154.

24 van den Oord JJ, Vandeghinste N, De Ley M, et al. Bcl-2 expression in human melanocytes and melanocytic tumors. Am J Pathol 1994;145:294-300.

25 Gould KL, Bretscher A, Esch FS, et al. cDNA cloning and sequencing of the protein-tyrosine kinase substrate, ezrin, reveals homology to band 4.1. EMBO J 1989;8:4133-4142.

26 Turunen O, Winqvist R, Pakkanen R, et al. Cytovillin, a microvillar $M_{\mathrm{r}} 75,000$ protein. cDNA sequence, prokaryotic expression, and chromosomal localization. J Biol Chem 1989;264:16727-16732.

27 Bretscher A, Reczek D, Berryman M. Ezrin: a protein requiring conformational activation to link microfilaments to the plasma membrane in the assembly of cell surface structures. J Cell Sci 1997;110:3011-3018.

28 Bretscher A, Edwards K, Fehon RG. ERM proteins and merlin: integrators at the cell cortex. Nat Rev Mol Cell Biol 2002;3:586-599.

29 Tsukita S, Yonemura S, Tsukita S. ERM (ezrin/radixin/ moesin) family: from cytoskeleton to signal transduction. Curr Opin Cell Biol 1997;9:70-75.

30 Vaheri A, Carpen O, Heiska L, et al. The ezrin protein family: membrane-cytoskeleton interactions and disease associations. Curr Opin Cell Biol 1997;9:659-666.

31 Bretscher A. Regulation of cortical structure by the ezrin-radixin-moesin protein family. Curr Opin Cell Biol 1999;11:109-116.

32 Turunen O, Wahlström T, Vaheri A. Ezrin has a COOHterminal actin-binding site that is conserved in the ezrin protein family. J Cell Biol 1994;126:1445-1453.

33 Gautreau A, Poullet P, Louvard D, et al. Ezrin, a plasma membrane-microfilament linker, signals cell survival through the phosphatidylinositol 3-kinase/Akt pathway. Proc Natl Acad Sci USA 1999;96:7300-7305.

34 Dransfield DT, Bradford AJ, Smith J, et al. Ezrin is a cyclic AMP-dependent protein kinase anchoring protein. EMBO J 1997;16:35-43.

35 Mackay DJ, Esch F, Furthmayr H, et al. Rho- and racdependent assembly of focal adhesion complexes and actin filaments in permeabilized fibroblasts: an essential role for ezrin/radixin/moesin proteins. J Cell Biol 1997;138:927-938.

36 Lugini L, Lozupone F, Matarrese $\mathrm{P}$, et al. Potent phagocytic activity discriminates metastatic and primary human malignant melanomas: a key role of ezrin. Lab Invest 2003;83:1555-1567.

37 Gutmann DH. Molecular insights into neurofibromatosis 2. Neurobiol Dis 1997;3:247-261.

38 Geiger KD, Stoldt P, Schlote W, et al. Ezrin immunoreactivity is associated with increasing malignancy of astrocytic tumours but is absent in oligodendrogliomas. Am J Pathol 2000;157:1785-1793.

$39 \mathrm{Yu} \mathrm{Y,} \mathrm{Khan} \mathrm{J,} \mathrm{Khanna} \mathrm{C,} \mathrm{et} \mathrm{al.} \mathrm{Expression} \mathrm{profiling}$ identifies cytoskeletal organizer ezrin and the developmental homeoprotein Six-1 as a key metastatic regulators. Nat Med 2004;10:175-181.

40 Khanna C, Wan X, Bose S, et al. The membranecytoskeleton linker ezrin is necessary for osteosarcoma metastasis. Nat Med 2004;10:182-186.

41 Moilanen J, Lassus $\mathrm{H}$, Leminen A, et al. Ezrin immunoreactivity in relation to survival in serous ovarian carcinoma patients. Gynecol Oncol 2003;90: 272-281.

42 Mäkitie T, Carpen O, Vaheri A, et al. Ezrin as a prognostic indicator and its relationship to tumour characteristics in uveal malignant melanoma. Invest Ophthalmol Vis Sci 2001;42:2422-2449.

43 Böhling $\mathrm{T}$, Turunen $\mathrm{O}$, Jääskeläinen J, et al. Ezrin expression in stromal cells of capillary hemangioblastoma. An immunohistochemical survey of brain tumours. Am J Pathol 1996;148:367-373.

44 Hunter KW. Ezrin, a key component in tumor metastasis. Trends Mol Med 2004;10:201-204.

45 Vo HP, Lee MK, Crowe DL. $\alpha 2 \beta 1$ integrin signaling via the mitogen activated protein kinase pathway modulated retinoic acid-dependent tumor cell invasion and transcriptional downregulation of matrix metalloproteinase 9 activity. Int J Oncol 1998;13: 1127-1134.

46 Orian-Rousseau V, Chen L, Sleeman JP, et al. CD44 is required for two consecutive steps in $\mathrm{HGF} / \mathrm{c}-\mathrm{Met}$ signaling. Genes Dev 2002;16:3074-3086.

47 Crepaldi T, Gautreau A, Comoglio PM, et al. Ezrin is an effector of hepatocyte growth factor-mediated migration and morphogenesis in epithelial cells. J Cell Biol 1997;138:423-434.

48 Recio JA, Merlino G. Hepatocyte growth factor/scatter factor induces feedback up-regulation of CD44v6 in melanoma cells through Egr-1. Cancer Res 2003;63: 1576-1582.

49 Cohen C, Zavala-Pompa A, Segueira JH, et al. Mitogenactivated protein kinase activation is an early event in melanoma progression. Clin Cancer Res 2002;8: 3728-3733.

50 Koo HM, VanBrocklin M, McWilliams MJ, et al. Apoptosis and melanogenesis in human melanoma cells induced by anthrax lethal factor inactivation of mitogen-activated protein kinase. Proc Natl Acad Sci USA 2002;99:3052-3057.

51 Collisson EA, De A, Suzuki H, et al. Treatment of metastatic melanoma with an orally available inhibitor of the Ras-Raf-MAPK cascade. Cancer Res 2003;63: 5669-5673.

52 Ichikawa T, Masumoto J, Kaneko M, et al. Moesin and CD44 expression in cutaneous melanocytic tumours. Br J Dermatol 1998;138:763-768.

53 Ohtani K, Sakamoto H, Rutherford T, et al. Ezrin, a membrane-cytoskeletal linking protein, is highly expressed in atypical endometrial hyperplasia and uterine endometrioid adenocarcinoma. Cancer Lett 2002;179:79-86.

54 Akisawa N, Nishimori I, Iwamura T, et al. High levels of ezrin expressed by human pancreatic adenocarcinoma cell lines with high metastatic potential. Biochem Biophys Res Commun 1999;258:395-400. 